\title{
Principais conceitos na implantação da sistemática de manutenção nas indústrias
}

\author{
Main concepts in the implementation of systematic maintenance in industries \\ Conceptos principales en la implementación del mantenimiento sistemático en industrias
}

Recebido: 12/12/2021 | Revisado: 18/12/2021 | Aceito: 26/12/2021 | Publicado: 05/01/2022

\author{
Tiago Rocha de Abreu \\ ORCID: https://orcid.org/0000-0002-5754-1989 \\ Universidade do Estado do Rio de Janeiro, Brasil \\ E-mail: tiago82871@hotmail.com
}

\begin{abstract}
Resumo
O aparecimento de equipamentos mais velozes e complexos mecanicamente, levam a adoção de atitudes, que visam sempre aumentar a produtividade e reduzir os custos da produção de qualquer tipo de produto. $\mathrm{O}$ dever da atividade de manutenção industrial na busca desses objetivos é um fator extremamente importante no sucesso e vida das empresas. Podemos expor a manutenção preventiva com um deles, evitando a ocorrência de falhas através de manutenções em intervalos de tempo regulares e pré-definidos. Tem como objetivo a eliminação ou redução, ao máximo, do número de falhas referentes à manutenção através de ações como limpeza, lubrificação, substituição de peças e verificação das instalações dos equipamentos, que sofrem esse tipo de intervenção partindo de um planejamento prévio das ações tomadas. Este trabalho, visa a apresentar os conceitos fundamentais da manutenção descrevendo-os e o efeito que a implantação de técnicas de manutenção causa sobre a produtividade das indústrias, com base nos conceitos de confiabilidade, disponibilidade e taxa de falhas. Com relação a metodologia, a pesquisa se caracteriza como integrativa buscando nas biografias os principais pontos importantes e necessários da área de manutenção aplicável às indústrias. Quanto aos métodos utilizados, o uso de vertentes conjuntas, têm mostrado extrema eficácia quando comparados a meios isolados de manutenção. A manutenção preditiva, embora na maior parte das ocasiões seja custosa, pode oferecer em conjunto com as demais vertentes uma melhoria nos indicadores de disponibilidade e performance dos equipamentos.
\end{abstract}

Palavras-chave: Manutenção preventiva; Disponibilidade; Confiabilidade; Falhas.

\begin{abstract}
The appearance of faster and more mechanically complex equipment has led to the adoption of attitudes that are always aimed at increasing productivity and reducing production costs for any type of product. The duty of industrial maintenance activity in pursuit of these goals is an extremely important factor in the success and life of companies. We can expose preventive maintenance with one of them, preventing the occurrence of failures through maintenance at regular and pre-defined time intervals. Its objective is to eliminate or reduce, as much as possible, the number of failures related to maintenance through actions such as cleaning, lubrication, replacement of parts and verification of equipment installations, which undergo this type of intervention based on prior planning of the actions taken. This work aims to present the fundamental concepts of maintenance describing them and the effect that the implementation of maintenance techniques has on the productivity of industries, based on the concepts of reliability, availability and failure rate. Regarding the methodology, the research is characterized as integrative, seeking in the biographies the main important and necessary points in the area of maintenance applicable to industries. As for the methods used, the use of joint strands has been shown to be extremely effective when compared to isolated means of maintenance. Predictive maintenance, although costly in most cases, can offer, together with the other aspects, an improvement in equipment availability and performance indicators.
\end{abstract}

Keywords: Preventive maintenance; Availability; Reliability; Failures.

\section{Resumen}

La aparición de equipos más rápidos y mecánicamente más complejos ha llevado a la adopción de actitudes que siempre tienen como objetivo aumentar la productividad y reducir los costos de producción de cualquier tipo de producto. El deber de la actividad de mantenimiento industrial en la consecución de estos objetivos es un factor de suma importancia en el éxito y la vida de las empresas. Podemos exponer el mantenimiento preventivo con uno de ellos, evitando la aparición de averías mediante un mantenimiento a intervalos regulares y predefinidos. Su objetivo es eliminar o reducir, en la medida de lo posible, el número de averías relacionadas con el mantenimiento mediante acciones como limpieza, lubricación, reposición de piezas y verificación de instalaciones de equipos, que se someten a este tipo de intervenciones en base a la planificación previa de las actuaciones tomado. Este trabajo tiene como objetivo presentar los conceptos fundamentales del mantenimiento describiéndolos y el efecto que la implementación de técnicas de mantenimiento tiene sobre la productividad de las industrias, con base en los conceptos de confiabilidad, disponibilidad y tasa de fallas. En cuanto a la metodología, la investigación se caracteriza por ser integradora, buscando en las biografías los principales 
puntos importantes y necesarios en el área de mantenimiento aplicable a las industrias. En cuanto a los métodos utilizados, el uso de cordones de unión ha demostrado ser extremadamente eficaz en comparación con los medios de mantenimiento aislados. El mantenimiento predictivo, aunque costoso en la mayoría de los casos, puede ofrecer, junto con los demás aspectos, una mejora en la disponibilidad de los equipos y en los indicadores de rendimiento.

Palabras clave: Mantenimiento preventivo; Disponibilidad; Fiabilidad; Fracasos.

\section{Introdução}

Através do desenvolvimento tecnológico, as máquinas tornaram-se cada vez mais rápidas, complexas e leves. Assim, houve a necessidade de desenvolver matérias primas com altos padrões de qualidade, operadores mais qualificados, tornando assim, extremamente necessária a manutenção eficiente e adequada desses equipamentos (Nepomuceno, 1989).

A manutenção se apresenta como fator importante no alcance da competitividade e do sucesso empresarial com a finalidade de oferecer o suporte a estratégia de produtiva, uma vez que a busca por novas ferramentas e técnicas de gestão dos seus ativos, que direcionem as empresas para uma maior competitividade nos quesitos de qualidade e produtividade, é o que as mantém num ciclo permanente de evolução (Pinto, 1999).

Embora a manutenção seja somente lembrada quando ocorrem falhas nos equipamentos ou instalações industriais, sendo mais especificamente quando um equipamento interrompe o seu funcionamento causando prejuízo muitas vezes financeiro, a manutenção sempre terá um essencial papel na produtividade das indústrias (Mobley, 2014).

Assim, é necessária a integração da atividade de manutenção ao processo produtivo das indústrias impulsionando-as diretamente ao caminho rumo a excelência. Por outro lado, fatores como a disponibilidade de máquina, a crescente competitividade, a busca pela lucratividade, satisfação do cliente, produtos isentos de defeitos, alta mantenabilidade, segurança dos trabalhadores e ambiente, estão diretamente ligados com a qualidade da manutenção empregada (Lafraia, 2001).

Tendo em vista todos os fatores citados acima, a atividade de manutenção não pode ser somente eficaz bastando apenas reparar o equipamento ou a instalação o mais rápido possível. A atividade de manutenção precisa principalmente manter o equipamento disponível operativamente, evitando a ocorrência de falhas e reduzindo possíveis riscos de uma interrupção de produção indesejado (Kardec \& Nascif, 2009).

\section{Metodologia}

A proposta desta etapa é desenvolver a estrutura teórica, com fundamentos em uma revisão da literatura, baseada em diversas publicações referentes ao assunto "manutenção". Serão descritos neste trabalho a conceituação e teoria da manutenção industrial, aspectos conceituais e os índices da manutenção, com o objetivo de dar consistência à construção do conhecimento e a base do presente estudo.

A técnica de pesquisa adotada para este artigo foi a revisão integrativa. A mesma tem o potencial de promover os estudos de revisão em diversas áreas do conhecimento, mantendo o rigor metodológico das revisões sistemáticas. O método de revisão integrativa permite a combinação de dados da literatura empírica e teórica que podem ser direcionados à definição de conceitos, identificação de lacunas nas áreas de estudos, revisão de teorias e análise metodológica dos estudos sobre um determinado tópico (Mendes, 2015).

Para este estudo foram selecionadas bibliografias que trouxessem em seu conteúdo conceitos fundamentais para a definição de "manutenção e suas derivações", bem como aplicações práticas e usuais de sua utilização nas indústrias. Foram selecionados artigos científicos, teses de graduações e livros de referência em universidades que possuíssem um amplo range de aplicação do conteúdo, compreendidas entre o período de 1989 à 2021, apresentando assim os conceitos base da manutenção e sua evolução ao longo de 30 anos de estudo. 
Para confeccionar esse artigo foi utilizado o guia “A Step-by-Step Guide to Conducting an Integrative Review” escrito por Toronto \& Remington (2020). Este guia é composto pelas seguintes etapas:

- Identificação do tema e seleção da questão da pesquisa

- Estabelecimento do critério de inclusão e exclusão

- Identificação dos estudos pré-selecionados e selecionados

- Categorização dos estudos selecionados

- Análise e interpretação de resultados

- Apresentação da revisão / síntese de conhecimento

\subsection{Definições}

Pode-se definir a manutenção como o conjunto de ações e técnicas administrativas destinadas a manter ou repor um determinado item em um estado no qual possa desempenhar uma determinada função (NBR 5462, 1994).

Consiste em medidas para efetuar a determinação e a avaliação da situação atual, bem como a preservação e reestabelecimento das condições normais de instalações, máquinas e componentes (Bertsche, 2008). Ela também é responsável em garantir a funcionalidade, conservação e controle da vida útil de equipamentos e instalações (Waeyenbergh, 2005).

A manutenção utiliza uma vasta gama de estratégias e ferramentas, as mesmas podem ser resumidas em políticas de manutenção. As políticas de manutenção são formadas por um conjunto de regras, conceitos e técnicas os quais são abordados para efetuar a operacionalização da manutenção de modo a atender os mais essenciais interesses industriais.

O Quadro 1 apresenta as principais políticas de manutenção e suas respectivas ações a serem tomadas.

Quadro 1 - Políticas de manutenção.

\begin{tabular}{|c|c|c|}
\hline \multicolumn{3}{|c|}{ Políticas de Manutenção } \\
\hline Grupo & Base da Politica de Manutenção & Ação da Manutenção \\
\hline Manutenção Corretiva & Manutenção Baseada na Falha & Reparar \\
\hline \multirow{3}{*}{ Manutenção Preventiva } & Manutenção Baseada no Uso & Inspecionar \\
\cline { 2 - 3 } & Manutenção Baseada no Tempo & Reparar \\
\cline { 2 - 3 } & Manutenção Baseada no Projeto & Substituir \\
\hline \multirow{2}{*}{ Manutençăo Preditiva } & Manutenção Baseada na Detecção & Inspecionar \\
\cline { 2 - 3 } & Manutenção Baseada na Condção & Inspecionar \\
\hline
\end{tabular}

Fonte: Adaptado de Waeyenbergh (2005).

A confiabilidade de um equipamento é afetada diretamente pela manutenção preventiva, resultando na diminuição na taxa de falhas ao logo do período de operação. Assim, de forma a elevar a produtividade anualizada de uma indústria, deseja-se que um equipamento esteja em operação e alcance uma taxa de utilização de uso imediato de 100\% (Lafrai, 2001).

Todavia, o parque industrial do Brasil é considerado obsoleto, tendo sua vida média de 15 à 25 anos e disponibilidade média em torno de $12 \%$ à $15 \%$. Isto significa uma disponibilidade duas vezes maior quando comparada a países desenvolvidos economicamente (Fuentes, 2006). Desta forma, o aumento da confiabilidade e disponibilidade das máquinas devem ser prioritários, uma vez que esses fatores estão relacionados diretamente com crescimento financeiro de uma indústria (Gruppi, 2006).

Os planos de manutenção preventiva são comumente utilizados para equipamentos e instalações fabris, têm o objetivo de garantir a funcionalidade dos processos e prevenir a ocorrência de possíveis falhas. Tais planos foram forjados através do tempo, 
com a observância dos históricos de falhas, através de orientações de fabricantes, inspeções "in loco" e através de melhorias para eliminar as causas-raízes (Corrêa, 2012).

Destaca-se que na utilização da manutenção na modalidade preventiva, a execução de intervenções programadas é antecipada, não ocasionando interrupções não planejadas da produção (Xenos, 1998, Contador, 1998).

Desta forma, a modalidade de manutenção preventiva é utilizada a mais de duas décadas pelas indústrias do Brasil, objetivando a melhoria de índices de manutenção dos equipamentos nos sites de produção, garantindo assim a qualidade esperada dos produtos e evitando falhas e/ou paradas da produção desnecessárias.

\subsection{Histórico da manutenção}

Por definição a manutenção é essencialmente a prestação de um serviço para um cliente interno da organização. Um serviço é qualquer ato ou desempenho, essencialmente intangível, que uma parte pode oferecer a outra, e que não resulta na propriedade de nada (Kotler e Keller, 2018, Zyrianoff, W., Kuniyoshi, M, Gaspar, M. \& Nascimento, H., 2021).

A manutenção está presente ao longo de eras da história da humanidade, ou seja, desde o momento em que iniciamos a manipulação de instrumentos produtivos. Os registros inicias da manutenção iniciam-se no século X, iniciando-se com o povo Viking que dependia fortemente da manutenção para manter seus poderosos navios prontos para a guerra a qualquer momento (Pascoli \& Silva, 2004). As primeiras menções ao termo "manutenção" são do século XII, mostrando o crescente avanço tecnológico da indústria (Arcuri Filho, 2005).

Porém, foi apenas com o desabrochar da Revolução Industrial do século XVIII, juntamente com o grande avanço da tecnologia, que a prática da manutenção surgiu nas indústrias, como meio de garantir a ininterrupção do trabalho. No início do século XX a manutenção era executada pelos próprios operários de produção, não tendo uma significativa importância (Tavares, 2000). Neste caso o próprio operário da máquina era responsável pela manutenção da mesma, recebendo assim treinamentos necessários para realizar reparos (Wyrebsk, 2007).

Esse cenário descrito acima permaneceu até meados da I Guerra Mundial, onde Henry Ford ao introduzir suas linhas de montagens iniciou a busca por uma sistemática de manutenção mais ágil e eficaz, sendo direcionada para o termo que hoje chamamos de manutenção corretiva (Filho, 2008).

Deste modo, o desenvolvimento tecnológico da manutenção acompanhou o desenvolvimento industrial por um centenário de história. Nas últimas três décadas as práticas da manutenção têm sofrido mais mudanças do que qualquer outra atividade, podendo, a partir da terceira década do século XX, serem distribuídas em quatro gerações (Kardec \& Nascif, 2009).

O Quadro 2 mostra a evolução da manutenção industrial descrevendo as características de cada geração. 
Quadro 2 - Evolução da manutenção industrial.

\begin{tabular}{|c|c|c|c|}
\hline $\begin{array}{l}\text { EVOLUÇÄO DA } \\
\text { MANUTENÇÄO }\end{array}$ & $\begin{array}{c}\text { EXPECTATIVAS QUANTO } \\
\text { AO DESEMPENHO DA } \\
\text { FUNCุÃO }\end{array}$ & $\begin{array}{c}\text { POLITICAS E } \\
\text { FILOSOFIAS } \\
\text { PREDOMINANTES }\end{array}$ & $\begin{array}{c}\text { TÉCNICAS E } \\
\text { PROCEDIMENTOS }\end{array}$ \\
\hline $\begin{array}{l}1^{\circ} \text { GERAÇÄO } \\
\text { (até 1940-1950) }\end{array}$ & $\begin{array}{c}\text { Reparo após avaria } \\
\text { Estabilidade da capacidade de } \\
\text { produçä̉o }\end{array}$ & Corretiva & $\begin{array}{l}\text { Substituição de Itens } \\
\text { Reparos de emergência } \\
\text { Isolamento da falha }\end{array}$ \\
\hline $\begin{array}{l}2^{\circ} \text { GERAÇÄO } \\
\text { (até 1950-1980) }\end{array}$ & $\begin{array}{c}\text { Maior Disponibilidade e } \\
\text { produtividade dos ativos físicos } \\
\text { Maior vida útil dos } \\
\text { equipamentos e componentes } \\
\text { Menores custos }\end{array}$ & Preventiva & $\begin{array}{c}\text { Troca sistematizada de } \\
\text { componentes } \\
\text { Revisões gerais } \\
\text { programadas } \\
\text { Sistemas de Planejamento e } \\
\text { controle }\end{array}$ \\
\hline $\begin{array}{l}3^{\circ} \text { GERAÇÄO } \\
\text { (até 1980-2000) }\end{array}$ & $\begin{array}{c}\text { Maior Disponibilidade e } \\
\text { confiabilidade dos ativos físicos } \\
\text { Maior vida útil dos } \\
\text { equipamentos e componentes } \\
\text { Maior segurança operacional } \\
\text { Maior produtividade, } \\
\text { competitividade e lucratividade. }\end{array}$ & $\begin{array}{c}\text { Preditiva } \\
\text { TPM } \\
\text { MCC (RCM) }\end{array}$ & $\begin{array}{c}\text { Monitoramento de condiçöes } \\
\text { e parâmetros operacionais } \\
\text { Inclusão da confiabilidade e } \\
\text { manutenibilidade nos } \\
\text { projetos } \\
\text { Análise de riscos, modos de } \\
\text { falhas, causas e efeitos. }\end{array}$ \\
\hline $\begin{array}{c}4^{\circ} \text { GERAÇÄO } \\
(2000-\ldots)\end{array}$ & $\begin{array}{l}\text { Alinhamento com os objetivos } \\
\text { estratégicos corporativos } \\
\text { Inserçäo nos sistemas } \\
\text { integrados de gestäo } \\
\text { Eng. De Manutençäo e } \\
\text { melhoria da manutenibilidade }\end{array}$ & $\begin{array}{c}\text { Pró-Ativa } \\
\text { Asset } \\
\text { Management } \\
\text { (Gestäo de Ativos } \\
\text { Físicos) }\end{array}$ & $\begin{array}{c}\text { Redes neurais } \\
\text { Sistemas especialistas } \\
\text { Interfaces "wireless" e "blue } \\
\text { tooth" } \\
\text { Multidisciplinaridade } \\
\text { Multiespecializacäd }\end{array}$ \\
\hline
\end{tabular}

Fonte: Adaptado de Moubray (2000).

\subsection{Custos de manutenção}

A periodicidade das ações de manutenção depende fortemente dos custos de manutenção envolvidos. É dita como a fundação para qualquer empresa que almeja lucro, também é essencial para a continuidade das ações de negócio.

Os custos de manutenção preventiva podem ser otimizados, a partir do controle do nível de degradação dos componentes, por meio da taxa de falha e consequentemente o ajuste da periodicidade das inspeções (Fouladirad, 2014). Desta forma a indústria do mundo todo gasta uma grande quantidade de dinheiro em manutenção das instalações (Loganathan, 2005).

Nos E.U.A., a três décadas atrás, as despesas de manutenção variavam entre $0,4 \%$ e $0,8 \%$ do total de vendas obtido, e no início do século XXI este nível subiu para 9\% à 15\% das vendas e de até $12 \%$ dos custos dos produtos comercializados (Kirby, 2002).

Atualmente há dois indicadores de extrema importância utilizados para a análise dos custos referentes à manutenção nas empresas: (Kardec \& Nascif, 2009)

- Custo de manutenção x lucro bruto da empresa (\%);

- Custo da manutenção x patrimônio da empresa (ativos pertencentes a uma empresa)

O C.B.M.G.A. - Congresso Brasileiro de Manutenção e Gestão de Ativos (2013), apresenta os custos gerados pela manutenção nas indústrias nacionais, possuindo um valor médio de 4,7\% do PIB referente ao ano em que foi realizada a pesquisa, conforme é mostrado na Tabela 1: 
Tabela 1 - Custo da manutenção no Brasil.

\begin{tabular}{|c|c|c|c|c|}
\hline \multicolumn{5}{|c|}{ CUSTO DA MANUTENÇĀO NO BRASIL } \\
\hline $\begin{array}{l}\text { Ano da } \\
\text { Pesquisa }\end{array}$ & $\begin{array}{l}\text { Ano } \\
\text { Base }\end{array}$ & $\begin{array}{c}\text { PIB } \\
\text { (Minhöes de R\$) }\end{array}$ & $\begin{array}{c}\text { CTMIFB } \\
{[\%]}\end{array}$ & $\begin{array}{l}\text { Custo } \\
\text { (Milhóes de RS) }\end{array}$ \\
\hline 2013 & 2012 & 4.403 .000 & 4,69 & $206.500,700$ \\
\hline 2011 & 2010 & 3.675 .000 & 3,95 & $145.162,500$ \\
\hline 2009 & 2008 & 2.900 .000 & 4,14 & $120.060,000$ \\
\hline 2007 & 2006 & 2.322 .000 & 3,89 & $90.325,800$ \\
\hline 2005 & 2004 & 1.769 .202 & 4,10 & $72.537,282$ \\
\hline 2003 & 2002 & 1.346 .028 & 4,27 & $57,475,396$ \\
\hline 2001 & 2000 & 1.101 .255 & 4,47 & $49.226,099$ \\
\hline 1999 & 1998 & 914.188 & 3,56 & $32.545,093$ \\
\hline 1997 & 1996 & 778.887 & 4,39 & $34,193,139$ \\
\hline 1995 & 1994 & 349.205 & 4,26 & $14.876,133$ \\
\hline
\end{tabular}

Fonte: Documento Nacional Abraman (2013).

Analisando a Tabela 1, nota-se que entre os anos de 1995 e 2011 a média é de aproximadamente 4,00\%, tendo ocorrido uma singela redução. Porém, após o ano de 2010, por causa de medidas governamentais que buscavam somente acelerar o crescimento do país, como o incentivo ao consumo e medidas protecionistas, culminaram em uma crise político econômica, ocasionando estagnação da economia, elevação da inflação e falta de investimentos no país.

Diante disto, ocorreu uma elevação percentual referente aos gastos de manutenção nas indústrias nacionais, ocasionando um disparo vertical nesses dados.

Permanecendo na esfera industrial, as despesas com manutenção representam na média um quinto (1/5) dos custos para se gerar um produto, sendo um ponto decisivo para a viabilização operacional de processos e máquinas.

No Brasil, considera-se a indisponibilidade como importante indicador na área de manutenção. Por definição é "a capacidade de um equipamento estar em condições de operação em um determinado período de tempo". Conforme verificado nas planas em atividade, esse indicador tem sofrido certa variação nos últimos 10 anos (NBR 5462, 1994). O Quadro 3 evidencia esse fato (Abraman, 2013). 
Quadro 3 - Indicadores de disponibilidade

\begin{tabular}{|l|c|c|c|c|c|c|c|c|c|}
\hline \multicolumn{1}{|c|}{ Indicadores de Disponibilidade } \\
\hline \multicolumn{1}{|c|}{ Tipo } & 1997 & 1999 & 2001 & $\mathbf{2 0 0 3}$ & $\mathbf{2 0 0 5}$ & $\mathbf{2 0 0 7}$ & $\mathbf{2 0 0 9}$ & $\mathbf{2 0 1 1}$ & $\mathbf{2 0 1 3}$ \\
\hline Disponibilidade Operacional & 85,82 & 89,30 & 91,36 & 89,48 & 88,20 & 90,82 & 90,27 & 91,30 & 89,29 \\
\hline Indisponibilidade devido a Manutençāo & 4,74 & 5,63 & 5,15 & 5,82 & 5,80 & 5,30 & 5,43 & 5,44 & 6,15 \\
\hline
\end{tabular}

Fonte: Documento Nacional Abraman (2013).

Constata-se pelo Quadro 3 que entre os anos de 1997 à 2013 a disponibilidade média era de aproximadamente $90 \%$, enquanto a indisponibilidade devida à manutenção teria valores próximos a 5,5\%. Observando estes índices, observa-se que ainda é possível realizar uma grande melhoria no planejamento e manutenção nas fábricas brasileiras, com o objetivo de melhorar a sua excelência.

\subsection{Tipos de manutenção}

O modo como é feito um reparo ou intervenção em uma máquina ou instalação fabril caracteriza seu respectivo tipo de manutenção. Existe uma enorme variedade de nomenclaturas que podem ser utilizadas para classificar os tipos de manutenção existentes. Como consequência essa variedade pode provocar certa confusão quando pretendemos caracterizar os tipos de manutenção (Kardec \& Nascif, 2009). Neste artigo, abordaremos somente três práticas básicas de manutenção, as quais são consideradas como principais por uma variedade de autores. O organograma da Figura 1, ilustra quais são essas.

Figura 1 - Organograma de manutenção.

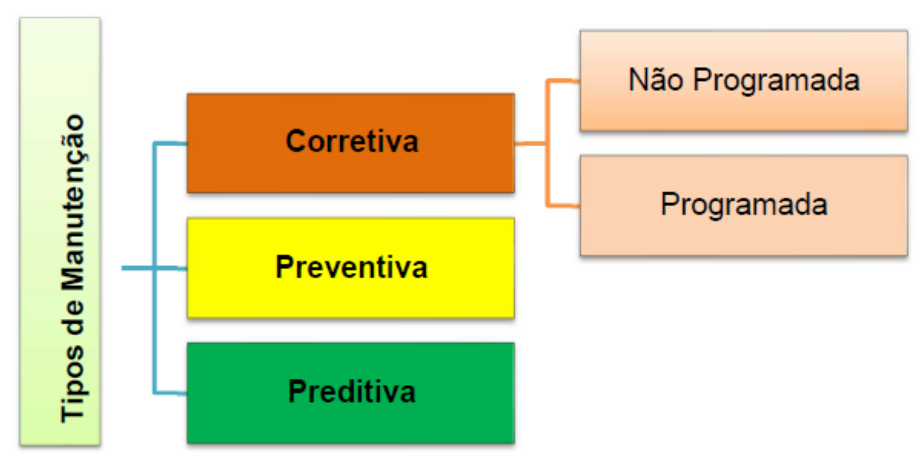

Fonte: Autor.

Antes de apresentar as principais características, vantagens, desvantagens e definições de cada tipo de manutenção, que serão introduzidas nos próximos tópicos deste artigo, temos no Quadro 4 uma breve apresentação das características das ações usualmente executadas nos diferentes tipos de manutenção pelos operadores responsáveis em uma instalação industrial.

Quadro 4 - Ações para os tipos de manutenção.

\begin{tabular}{|c|c|}
\hline Manutenção & Ação \\
\hline Corretiva não programada & Após a ocorrência, sem planejamento \\
\hline Corretiva planejada & Planejada, função de inspeção ou acompanhamentos preditivos \\
\hline Preventiva & Planejada, com intervalos definidos em plano \\
\hline Preditiva & Inspeção/ Acompanhamentos de Parâmetros Físicos \\
\hline
\end{tabular}

Fonte: Autor. 


\subsubsection{Manutenção corretiva}

A manutenção corretiva caracteriza-se pela execução das ações após o surgimento de uma falha ou defeito em um equipamento. Tem como objetivo restaurar um equipamento à condição anterior a falha de forma que o mesmo venha executar uma função requerida (NBR 5462, 1994).

Este tipo de manutenção, considerada simples, exige altos custos referentes a "spare parts - partes de reposição", horas extras dos operários, ociosidade do equipamento e a baixa disponibilidade de produção (Almeida, 2000). Somado a este ponto, temos o fato dos custos tenderem a aumentar rapidamente caso o tempo para se agir e reparar um equipamento se prolongue, seja por fatores como indisponibilidade de mão de obra qualificada ou escassez de peças de reposição.

Sendo assim, pode-se dividir a manutenção corretiva em duas classes:

- Não planejada: efetua a correção das falhas de modo aleatório, como uma quebra inesperada, sempre após ocorrer o fato e sem gerenciamento anterior. Caso o reparo ou uma quebra não possam ser postergados, devem ser considerados como uma manutenção emergencial ou corretiva não planejada, ou seja, ao aparecer a falha é necessário o reparo imediato da mesma (Branco, 2006). Nesta modalidade ao ser notada a parada do equipamento, a equipe de manutenção é mobilizada para o reparo imediato do equipamento.

- Planejada: é uma correção de uma falha ou de um desempenho menor que o esperado, porém se baseia na modificação dos parâmetros de condição observados pela manutenção preditiva (Kardec \& Nascif, 2009). Tem como principal diferença da manutenção não planejada ser uma decisão gerencial. Desta forma, a empresa ao implantar o sistema de manutenção planejada obtém algumas vantagens, como: (Kardec e Nascif, 2009)

- Possibilita o agendamento da manutenção em comum acordo com a produção;

- Aumento da segurança, já que a falha provoca riscos para o pessoal e instalação;

- Melhor planejamento dos serviços;

- Garante a existência de peças sobressalentes, equipamentos e ferramental;

- Facilita a organização da equipe de trabalho, aquisição de tecnologias e outros recursos se necessários, que podem inclusive, serem buscados externamente à organização.

\subsubsection{Manutenção preventiva}

Define-se a manutenção preventiva como aquela em que as ações são executadas em períodos predeterminados, conforme orientações prescritas e planejadas, com o objetivo de reduzir as chances de ocorrência de falhas ou o decaimento do desempenho de um equipamento (Lafraia, 2001). Tem como objetivo preservar o equipamento, seguindo ações periódicas regulares de manutenção, seguindo o manual de manutenção fornecido pela fabricante (Aquino, E., Neto, M, Bernardo, C., Morais, F. \& Santos, P., 2020).

A manutenção preventiva utiliza muitas vezes meios técnicos para estimar o momento em que ocorrerá a falha no equipamento, sendo esses meios muitas vezes instrumentos de medições para falhas específicas, ou parecer técnico de um especialista que contenha conhecimento amplo nesta área (Garg \& Deshmukh, 2006, Pieretti, R., Silva, M, Lesme, D. \& Almeida, M. (2020).

Ao optar pela implementação do sistema de manutenção preventivo, este deve ser de caráter obrigatório pois possui a intenção de intervir em um equipamento antes que ocorra a sua quebra (Xenos, 1998).

Utilizando este método de manutenção, os períodos em que são realizadas as intervenções são programados antecipadamente, ou seja, não ocorre interrupção inesperada da produção (Contador, 1998). 
Esse método de manutenção é bastante planejado oferecendo uma lista enorme de vantagens. Abaixo seguem alguns pontos positivos a respeito da manutenção preventiva: (Monchy, 1989).

- Aumenta a confiabilidade de um equipamento e assim reduz suas falhas em operação: reduz os custos de falha e melhora a disponibilidade:

- Aumenta a duração da vida eficaz de um equipamento;

- Melhora o planejamento dos trabalhos, e assim, as relações com a produção;

- Reduz e regulariza a carga de trabalho da manutenção;

- Facilita a gerência dos estoques (consumos previstos);

- Aumenta a segurança (menos improvisações perigosas);

- Reduz a "surpresa", melhorando o clima das relações humanas (uma pane sempre gera tensões).

Uma exigência para a implementação da manutenção preventiva é ter-se um almoxarifado de peças enxuto e o mais eficiente possível, ou seja, ter em estoque somente as peças necessárias para manter uma máquina em perfeito funcionamento e usá-las no momento certo.

\subsubsection{Manutenção Preditiva}

É o método de manutenção que requer acompanhamento de várias variáveis e parâmetros de desempenho dos equipamentos, tendo como objetivo estimar o instante exato da intervenção com aproveitamento máximo do ativo (Otani \& Machado, 2008).

A manutenção preditiva tem o objetivo de monitorar e/ou acompanhar os equipamentos e peças, por medições através de métodos específicos ou controle estatístico, deste modo é possível fazer a predição da falha com maior precisão (Viana, 2014). Conforme o autor citado, este tipo de manutenção tem como objetivo determinar o momento exato da intervenção, assim, aproveitando ao máximo a vida útil do equipamento ou peça ao máximo.

Atualmente as quatro técnicas de manutenção preditiva mais utilizadas nas indústrias nacionais são: ensaio por ultrassom; análise de vibrações mecânicas, análise de óleos lubrificantes e termografia.

\section{Resultados e Discussão}

\subsection{Conceitos aplicados: Tagueamento}

A palavra "Tag" na língua inglesa significa "etiqueta de identificação" e nas indústrias o ato de identificar equipamentos com etiquetas significa "tagueamento" (Viana, 2014). O tagueamento tem como finalidade cadastrar e identificar cada tipo de equipamento na sua exata localização dentro de uma instalação industrial.

A implantação do tagueamento nas indústrias possibilita um controle exato de todas as manutenções efetuadas em um equipamento, no planejamento de serviços, na obtenção de indicadores mais confiáveis para cada equipamento e a segurança dos operadores que estão realizando o serviço de manutenção, de forma que não ocorra equívocos em relação a máquina a ser tratada.

Com a implementação do sistema de tagueamento, é possível efetuar a criação de um banco de informações contendo o histórico de manutenções efetuadas para cada máquina para assim ser feito o total gerenciamento sobre este ativo. Pode-se afirmar que esse sistema é de extrema importância para a área de Planejamento e Controle de Manutenção (PCM). 


\subsection{Conceitos aplicados: Banco de dados}

Banco de dados é uma estrutura bem organizada, composta de coleções de informações de dados, que se relacionam de forma que crie um sentido e permita a extração de informações. Ou seja, consiste de um conjunto informações referentes a manutenção, pessoal, serviços, eventos e ocorrências.

\subsection{Conceitos aplicados: Ordem de serviços (O.S)}

A Ordem de serviço (O.S) é um detalhamento escrito, enviado fisicamente ou digitalmente, que define as ações a serem tomadas em relação a um equipamento (Viana, 2014). É por meio desse documento que é realizada a formalização de uma intervenção a ser feito entro de uma indústria pelo gerente ou gestor de manutenção. A O.S. é seguida de uma C.I. (comunicação interna) para avisar aos mantenedores, sobre a necessidade de realização de uma tarefa específica.

O documento físico ou manual possui informações as quais dependem das atividades que são feitas na empresa. Podemos destacar os elementos de uma O.S. como: dados do local do serviço a ser realizado, número de processamento, data de emissão e assinatura do emissor juntamente com seu respectivo cargo.

A O.S. possui uma codificação, geralmente um número sequencial, que tem como objetivo diferenciar uma ordem de serviço de outras demais, de forma que a área mantenedora possa organizar os trabalhos a serem realizados e executar a priorização dos mesmos.

Além dos dados citados acima, a O.S pode carregar em seu corpo a discriminação das tarefas a serem a executadas bem como respectivas recomendações, procedimentos e ferramentas a serem utilizados, facilitando a execução da tarefa.

\subsection{Conceitos aplicados: Falhas}

Define-se falha como "o fim da capacidade de um equipamento executar a função ao qual foi projetado" (NBR 5462, 1994). É caracterizada pela perda de função plena de um equipamento. As falhas podem ser agrupadas em duas classes, conforme o grau de redução de capacidade de funcionamento: (Nascif, 2009)

\section{- Falhas Totais}

- Falhas Parciais

As falhas parciais são caracterizadas pela capacidade do equipamento operar durante um período de tempo, mesmo que a falha já tenha ocorrido. Podemos citar como exemplo de falha parcial um rolamento defeituoso. As falhas totais são caracterizadas quando não há capacidade ou possibilidade de um equipamento funcionar após a ocorrência da falha. Podemos citar como exemplo de falha parcial a quebra de um eixo de um caminhão.

Podemos classificar, também, as falhas conforme o critério de evolução no tempo:

- Falhas Catastróficas

- Falhas Graduais

Falhas catastróficas, podemos exemplificar como rompimento de uma linha férrea ou quebra de uma pá de uma turbina a gás. O desgaste do diâmetro de um mancal pode ser exemplificado como uma falha gradual.

Alguns segmentos industriais classificam as falhas quanto a sua duração, conforme abaixo: (Viana, 2014)

- Falhas Temporárias (superaquecimento de um motor de combustão devido ausência de fluído refrigerante);

- Falhas Intermitentes (falta de contato de uma bateria no interior de uma lanterna);

- Falhas Permanentes (fratura de uma guia de suporte de um equipamento que esteja submetido a vibração);

Uma outra classificação que pode ser feita é referente a relação de falhas entre si, conforme abaxo:

- Dependente

- Independente 
A falha dependente em um componente induz a falha em outros, já a falha independente ocorre sem nenhuma relação intrínseca com outro componente

\section{Considerações Finais}

Recomenda-se às indústrias para não medirem esforços para implantar planos de manutenção preventiva suas máquinas, das suas linhas de produção, a fim de melhorar a produção, diminuir o número de paradas na produção e redução nos custos nas manutenções corretivas emergenciais.

Sugere-se também que se invista em práticas de manutenção preditiva para que possa ser feito em conjunto com as manutenções preventiva e assim, melhorar ainda mais os indicadores analisados.

Dessa forma, para implementação da sistemática de manutenção nas indústrias, recomenda-se utilizar índices de desempenho da manutenção que explorem o âmbito econômico, ou seja, o custo que a manutenção preventiva tem frente a manutenção corretiva, para que os resultados possam justificar o investimento inicial necessário para a implantação da manutenção preventiva.

Para trabalhos futuros, recomenda-se a análise mais aprofundada de cada tipo de manutenção apresentado bem como as interações entre si. Isto poderá gerar mais artigos e pesquisas como, por exemplo, o estudo da viabilidade financeira para implementação nas rotinas de manutenção preditiva, em conjunto com a manutenção preventiva, para equipamentos fabris.

\section{Referências}

ABNT - Associação Brasileira de Normas Técnicas. (1994). NBR 5462/1994 - Confiabilidade e Mantenabilidade. Rio de Janeiro: Copyright.

Abraman - Associação Brasileira de Manutenção. Documento Nacional (2013). A situação da manutenção no Brasil. In: Congresso Brasileiro de Manutenção, 20013, Bahia. Anais. Salvador.

Almeida, M. T. (2000). Manutenção Preditiva: Confiabilidade e Qualidade. http://www.mtarv.com.br/download/mnt1.pdf.

Aquino, E., Neto, M., Bernardo, C., Morais, F. \& Santos, P. (2020). Ferramentas de manutenção preditiva de motores diesel: uma revisão bibliográfica sistemática. Research, Society and Development, 9 (11), p.4, e57691110195.

Arcuri-Filho, Rogério \& Carvalho, N.(2005). Medicina de Sistemas: o futuro conceito de Manutenção. Revista de Ensino de Engenharia, Rio de Janeiro Brasil, n.12, p.11-17.

Bertsche, B. (2008). Confiabilidade em Engenharia Automotiva e Mecânica: Determinação da Confiabilidade de Componentes e Sistemas. Berlim: Springer.

Branco Filho, Gil. (2006). Indicadores e Índices de Manutenção. Rio de Janeiro: Ciência moderna Ltda.

Contador J. C et al. (1998). Gestão de Operações: a engenharia de produção a serviço da modernização da empresa. 2. ed. São Paulo: Edgard Blucher LTDA.

Corrêa, H. L. \& Corrêa, C. (2008). Administração da produção e operações: Manufatura e serviços: uma abordagem estratégica. 2. ed. São Paulo: Atlas.

Filho, R. (2008). A Introdução à Manutenção Centrada na Confiabilidade - MCC. Programa de Atualização Técnica 2008 - Sistema FIRJAN - SESI/SENAI Rio de Janeiro.

Fouladirad M., grall. (2014). Detecção de mudanças on-line e manutenção baseada em condições para sistemas com parâmetros de deterioração desconhecidos. IMA Journal of Management Mathematics. Oxford University Press, v. 25, p.139-158.

Fuentes, F. (2006). Metodologia para inovação da gestão de manutenção industrial. Tese (Doutorado) - Curso de Engenharia Mecânica, Universidade Federal de Santa Catarina, Florianopólis.

Garg, A., \& Deshmukh, S. G. (2006). Maintenance management: literature review and directions. Journal of quality in maintenance engineering. 13 (06), p.218, $10.1108 / 13552510610685075$.

Gruppi, G. (2006). Estrutura de manutenção na indústria automobilistica: uma análise comparativa dos modelos especialista e generalista. 137 f. Dissertação (Mestrado) - Curso de Sistema de Gestão, Centro Tecnológico, Universidade Federal Fluminense, Niteroi.

Kardec, A., Nascif, J. (2009). Manutenção: Função estratégica. 3. ed. Rio de Janeiro: Qualitymark.

Kirby, E. (2002). Há ouro nessas práticas de confiabilidade e manutenção. 1. ed. Rio de Janeiro: Atlas.

Kotler, P. \& Keller, K. (2018). Administração de marketing. Prentice Hall. 
Lafraia, J. R. B. (2001). Manual de confiabilidade, mantenabilidade e disponibilidade. Rio de Janeiro: Editora Qualitymark Ltda.

Loganathan, K., Faisal, K., Mahmoud H. (2005). Desenvolvimento de uma estratégia de manutenção baseada em riscos (RBM) para uma usina de geração de energia. Jornal de Prevenção de Perdas [Indústrias de Processos]. v. 18, p.69-81.

Mendes, K.(2015). Tipos de revisão de literatura. https://www.fca.unesp.br/Home/Biblioteca/tipos-de-evisao-de-literatura.pdf

Mobley, R. K., Higgins, L. R., \& Wikoff, D. J. (2014). Manual de Engenharia de Manutenção, McGrawhill. 8 ed. Nova Iorque, Chicago, São Francisco, Lisboa, Londres, Madrid, Cidade do México, Milão, Nova Deli, San Juan, Seul, Singapura, Sydney e Toronto. Impresso pelos EUA.

Mochy, F. (1989). A função manutenção. 1. Ed. São Paulo: Ebras Durban.

Nepomuceno, L. X. (1989). Técnicas de Manutenção Preventiva. São Paulo: Edgard Blucher Ltda.

Otani, M. \& Machado, W. V. (2008). A proposta de desenvolvimento de gestão da manutenção industrial na busca da excelência ou classe mundial. Revista Gestão Industrial. 4(2),1-16.

Pascoli, J. \& Silva, R. (2004). Gerenciamento do setor de manutenção. Trabalho de conclusão do curso para obtenção do certificado de especialização em gestão Industrial do departamento de economia, contabilidade, Administração da Universidade de Taubaté.

Pieretti, R., Silva, M, Lesme, D. \& Almeida, M. (2020). Análise de indicadores de desempenho individual aplicado a manutenção industrial. Research, Society and Development, 9 (6), 6, e129963660.

Pinto, A. \& Nascif, J. (1999). Manutenção: Função Estratégica. Rio de Janeiro: Qualitymark.

Tavares, L. (2000). Administração Moderna e Manutenção. 1 edição. Rio de Janeiro: Novo Pólo.

Toronto, E. \& Remington, R. (2020). A Step-by-Step Guide to Conducting an Integrative Review. Massachusetts: Srpinger.

Viana, H. (2014). PCM, Planejamento e Controle da Manutenção. Rio de Janeiro: Qualitymark.

Xenos, H. (1998). Gerenciando a Manutenção Preventiva: o caminho para eliminar falhas nos equipamentos e aumentar a produtividade. Belo Horizonte: Editora de Desenvolvimento Gerencial.

Waeyenbergh, G. (2005). Uma estrutura para o desenvolvimento do conceito de manutenção industrial. Leuven, Belgica: Katholieke Universiteit Leuven.

Wyrebsk, J. (2018). Manutenção Produtiva Total. Um Modelo Adaptado. Dissertação (Mestrado em Engenharia de Produção). Universidade Federal de Santa Catarina, Florianópolis.

Zyrianoff, W., Kuniyoshi, M, Gaspar, M. \& Nascimento, H. (2021). Práticas de Gestão do Conhecimento e Capacidade Absortiva Aplicadas à Melhoria de Desempenho e Qualidade na Manutenção Industrial. Research, Society and Development, 10 (2), 8, e47410212713. 